Pp. 1-8

DOI: $10.1089 /$ cap.2018.0060

\title{
Use of Nutritional Supplements in Youth with Medicated and Unmedicated Attention-Deficit/Hyperactivity Disorder
}

\author{
Oliver Scholle, MSc, ${ }^{1}$ Hannah Jilani, MPH, ${ }^{2}$ Oliver Riedel, PhD, ${ }^{1}$ Tobias Banaschewski, MD, PhD, ${ }^{3}$ \\ Charalambos Hadjigeorgiou, $\mathrm{PhD},{ }^{4}$ Monica Hunsberger, $\mathrm{PhD},{ }^{5}$ Isabel Iguacel, MSc, ${ }^{6}$ \\ Dénes Molnár, PhD, ${ }^{7}$ Valeria Pala, Dr Sc, ${ }^{8}$ Paola Russo, MSc, ${ }^{9}$ Toomas Veidebaum, PhD, ${ }^{10}$ \\ Mahmoud Zaqout, MD, ${ }^{11}$ and Hermann Pohlabeln, $\mathrm{PhD}^{12}$; on behalf of the I.Family Consortium
}

\begin{abstract}
Objective: To find out whether use of nutritional supplements (NUS) differs between children and adolescents with attentiondeficit/hyperactivity disorder (ADHD; medicated or unmedicated), compared with those without the disorder.

Methods: We used cross-sectional data from the population-based I.Family study conducted between 2013 and 2014 in eight European countries. Parents completed questionnaires and participated in interviews, for example, on health and medical history of their child. Data from 5067 children and adolescents aged 5-17 years were included. Exposures were medicated (with ADHD-approved medication) and unmedicated ADHD. The outcome was the use of NUS, measured by use of any or multiple different NUS. Multivariable logistic regression adjusted for sociodemographics and health determinants was used to find ADHD-depending differences.

Results: The study sample comprised 4490 children and adolescents without ADHD and 51 medicated and 76 unmedicated subjects with ADHD. Regarding the use of any NUS, no statistically significant differences were found between children and adolescents without ADHD (18\%) and those with medicated (18\%) or unmedicated ADHD (22\%). However, discrepancies appear when considering multiple use of NUS, not reported for any medicated ADHD subject but remarkably often for unmedicated ADHD subjects (13\%), resulting in an adjusted odds ratio of 2.6 (95\% confidence interval, 1.2-5.6) when compared with those without ADHD (5\%).

Conclusion: Children and adolescents who were not using medication for treating ADHD potentially took NUS as oral remedies. Given the potential for a delay of indicated treatments and for use of those NUS which have no proven effectiveness, pediatricians should actively explore whether NUS have been used to treat ADHD core symptoms, and families should be informed that the average effect size has to be considered small.
\end{abstract}

Keywords: attention deficit disorder with hyperactivity, dietary supplements, child, adolescent, drug therapy

Departments of ${ }^{1}$ Clinical Epidemiology and ${ }^{2}$ Epidemiological Methods and Etiologic Research, Leibniz Institute for Prevention Research and Epidemiology—BIPS, Bremen, Germany.

${ }^{3}$ Department of Child and Adolescent Psychiatry and Psychotherapy, Central Institute of Mental Health, Medical Faculty Mannheim/Heidelberg University, Mannheim, Germany.

${ }^{4}$ Research and Education Institute of Child Health, Strovolos, Cyprus.

${ }^{5}$ Section for Epidemiology and Social Medicine (EPSO), Institute of Medicine, The Sahlgrenska Academy at University of Gothenburg, Gothenburg, Sweden.

${ }^{6}$ GENUD (Growth, Exercise, NUtrition and Development) Research Group, Faculty of Health Sciences, University of Zaragoza, Zaragoza, Spain.

${ }^{7}$ Department of Paediatrics, Medical School, University of Pécs, Pécs, Hungary.

${ }^{8}$ Epidemiology and Prevention Unit, Fondazione IRCCS Istituto Nazionale dei Tumori, Milan, Italy.

${ }^{9}$ Unit of Epidemiology and Population Genetics, Institute of Food Sciences, National Research Council, Avellino, Italy.

${ }^{10}$ Department of Chronic Diseases, National Institute for Health Development, Tallinn, Estonia.

${ }^{11}$ Department of Public Health, Faculty of Medicine and Health Sciences, Ghent University, Ghent, Belgium.

${ }^{12}$ Department of Biometry and Data Management, Leibniz Institute for Prevention Research and Epidemiology-BIPS, Bremen, Germany.

Funding: The I.Family Study was financially supported by the European Commission within the Seventh RTD Framework Programme Contract number 266044. Additional resources were invested by all participating partners. The current study received no specific funding. No funder had any role in the study design, the collection, analysis, and interpretation of data, the writing of the article, and the decision to submit it for publication. 


\section{Introduction}

A TTENTION-DEFICIT/HYPERACTIVITY DISORDER (ADHD) is a common neurodevelopmental disorder with a worldwide prevalence of $3.4 \%$ in children and adolescents (Polanczyk et al. 2015). Apart from pharmacological treatments with stimulants, nutritional supplements (NUS), categorized as "complementary and alternative treatments," are also commonly used (Chan et al. 2003; Sinha and Efron 2005). Until recently, clinical guidelines on ADHD either did not explicitly mention complementary and alternative medicine, such as NUS, or reported a lack of evidence for their use (Taylor et al. 2004; Pliszka and AACAP Work Group on Quality Issues 2007; Subcommittee on Attention-Deficit/ Hyperactivity Disorder et al. 2011).

However, since 2016, the ADHD guideline by the British National Institute for Health and Care Excellence (NICE) clearly has advised against use of dietary polyunsaturated fatty acid supplementation for treating ADHD (NICE 2018). Research published after this update of the NICE guideline suggests that polyunsaturated fatty acids — particularly omega-3 fatty acids from fish oil — might be effective in reducing the dosage of the ADHD medication (Königs and Kiliaan 2016; Lange et al. 2017). In addition, zinc, iron, and magnesium might assist in alleviating ADHD symptoms in children with deficiencies in any of these minerals or with an increased risk thereof (Bloch and Mulqueen 2014; Lange et al. 2017).

Also, results from a randomized controlled trial indicate that children with ADHD and comorbid emotional dysregulation may benefit from certain vitamin/mineral supplementation by improving emotional regulation, aggression, and general functioning (Rucklidge et al. 2018). Nevertheless, more studies in this area are needed before the efficacy of these supplements can be properly measured.

The lack of evidence is contrary to the high popularity of complementary and alternative medicine indicated by previous studies. At the end of the 1990s, more than half of 114 U.S. parents of children with ADHD in one clinic had reported using complementary and alternative medicine for their child during the past year (Chan et al. 2003) and in 2003, about two-thirds of parents in Australia reported ever having used complementary and alternative medicine to treat their ADHD-affected children (Sinha and Efron 2005). NUS were among the most common interventions used. However, these studies did not have a comparison group and used clinical samples usually representing more severely affected ADHD patients.

Results from the 1999 to 2002 U.S. National Health and Nutrition Examination Surveys (NHANES) indicated that adolescents with ADHD were more likely to use NUS than those without ADHD (Gardiner et al. 2008). Use of NUS was generally more common in those who used prescription drugs. As the study did not focus on ADHD patients, the influence of using ADHD medication on the results remained unclear. Furthermore, only participants aged 11 years and older were included. That age is beyond the typical ADHD onset and differences might be more pronounced when younger children are also included.

Moreover, most evidence regarding fish oil as an omega-3containing NUS to treat ADHD only emerged starting in the mid2000s (Heilskov Rytter et al. 2015). In addition, advertisements broadly formulating the beneficial effects of such NUS on ADHD symptoms might have led to an increased use since the publication of the NHANES data. In fact, use of omega-3 fatty acid supplements among U.S. children and adolescents increased between 2003 to 2004 and 2013 to 2014 from $0.4 \%$ to 2.3\% (Qato et al. 2018).

In this study, we aimed to assess differences in use of NUS among children and adolescents with ADHD compared with those without the disorder in a population-based sample. The research question was stimulated by the results of the NHANES study, where adolescents with ADHD had a slightly higher (10\%) use of any NUS than other adolescents (Gardiner et al. 2008). The study also showed that adolescents who used prescription drugs were significantly more likely (37\%) to use any NUS (Gardiner et al. 2008). Compared with the NHANES study, our data enabled us to estimate the effect of use of prescription drugs without considering medication to treat ADHD. Furthermore, they allowed us to ask a more specific research question: Is the use of NUS in children and adolescents with ADHD dependent on whether they are treated with ADHD medication or not?

\section{Methods}

\section{Setting}

Data were used from the European I.Family study conducted between 2013 and 2014 in eight countries (Belgium, Cyprus, Estonia, Germany, Hungary, Italy, Spain, and Sweden). The study can be regarded as the second follow-up examination of a community-based field study on "Identification and prevention of Dietary- and lifestyle-induced health Effects In Children and infantS" (IDEFICS) (Ahrens et al. 2011). Examination modules comprised several questionnaires, physical examinations, and biological samples. The cohort profile has been described in detail elsewhere (Ahrens et al. 2017).

\section{Ethical considerations}

Each participating center obtained ethical approval from the local responsible authorities. Informed consent from the children and their parents was required before any data collection and they were free to reject single examination modules and questionnaires.

\section{Study design and recruitment}

An initial baseline examination (T0) in the context of the abovementioned IDEFICS study - the data of which were not used for this study as use of NUS was not recorded-took place from 2007 to 2008, followed by a first follow-up examination (T1) from 2009 to 2010, in at least two local not randomly selected communities per country. All children aged 2-9 years attending kindergarten and elementary school had been eligible. As part of the subsequent I.Family study-which provided the data for this study - children who participated in T0 and/or T1 of IDEFICS (hereafter referred to as index children), as well as their parents and siblings, were invited for a second follow-up. Thus, between 2013 and 2014, data were provided from 6167 families. For the present cross-sectional analysis, all 5- to 17-year olds were eligible and we selected one member from each family using an algorithm described under the "Exclusion Criteria" section.

General information about the respondent or the family such as sociodemographic characteristics was given by at least one parent or legal guardian. Information on the medical history of the participants, including diseases, drug use, and the intake of NUS, was obtained from at least one parent living in the main household using a computer-assisted personal or telephone interview.

\section{Study measures}

Participants were categorized as having ADHD depending on a parent-reported diagnosis and/or use of any of the following ADHD-approved medications: methylphenidate, atomoxetine, 
amphetamine, dexamphetamine, lisdexamfetamine. Centrally acting alpha-agonists (clonidine or guanfacine) were not approved for ADHD in Europe during the study years. Details about the diagnosis, drug use, and use of NUS within the last 14 days were collected using a questionnaire about chronic diseases diagnosed in the individual participant. A subject with ADHD was categorized as "medicated" if at least one of the abovementioned ADHDapproved medications had been used.

The study outcome was use of NUS, measured by use of (1) any or (2) multiple different NUS (either simultaneously or sequentially) during the last 14 days. The measure regarding multiple different NUS reflecting a more intensive intake was added post hoc to the protocol during the analysis, since we observed conspicuously high rates of the differently classified NUS (e.g., omega3 fatty acids and vitamin D) in unmedicated subjects with ADHD. In addition to given categories of NUS, parents could specify additional products, which interviewers entered as free text. Free-text records were allocated to the respective categories; for example, brand names from omega-3 products were assigned to "omega-3 fatty acids." A pharmacist carried out the allocation using blinded records, that is, without information on the ADHD diagnosis. This was reviewed by one person from each participating country.

As covariates, we included characteristics shown by former studies to be either associated with ADHD and/or use of NUS or those hypothesized to be associated with any form of dietary intervention (e.g., food intolerance). Use of prescription drugs (other than ADHD medications) during the last 14 days was assessed from free-text records on medication that were processed as described for the NUS. The following parent-reported chronic diseases of the child were included: food intolerance (e.g., to lactose, fish and crustaceans, fruits and vegetables), serious infection requiring hospitalization, and allergic diseases (e.g., eczema and hay fever).

Regarding parental psychiatric disorders, the interviewer asked about psychological/psychiatric problems. The highest parental education level was coded according to the 2011 International Standard Classification of Education and further categorized into low (0-2), medium (3-5), and high (6-8) (United Nations Educational, Scientific and Cultural Organization 2011). Income level was categorized into five groups by linking the income to the average country-specific net household income.

\section{Exclusion criteria}

Subjects were excluded from the analysis if a completed questionnaire on health and medical history was not available. Moreover, to avoid any effects of familial clustering, we excluded siblings using the following algorithm: participants who had been diagnosed with ADHD were identified first and their siblings without ADHD excluded. From families without any or more than one ADHD subject, nonindex children were excluded. If more than one index child was eligible, we randomly selected one.

\section{Statistical analyses}

Using descriptive statistics, sample characteristics and use of NUS were shown for each of the three groups, namely children and adolescents without ADHD (1) and those with ADHD who were unmedicated (2) or medicated (3). Two multivariable logistic regressions were used to identify group-dependent differences: first, a partially adjusted model, controlling for the demographic variables age, sex, and country, and second, a fully adjusted model, taking also into account education level, income level, serious infections, allergic diseases, food intolerance, and parental psychiatric disorders.
When considering education and income level as confounders, missing values for these variables were treated as separate categories and taken into account as dummy variables in the regression models.

An additional analysis was conducted to compare all subjects with ADHD - independently of medication status-with non-ADHD peers to evaluate the comparability of our estimates (fully adjusted) with those obtained from the NHANES data. In addition, although parent-reported ADHD diagnoses have shown convergent validity (Visser et al. 2013), we attempted to corroborate the parent-reported ADHD diagnoses in our study. Using the $t$-test, we compared subjects with and without ADHD regarding mean differences in a score for impulsiveness based on survey items. Higher scores indicate more impulsive behavior of a subject according to the negative urgency subscale of the UPPS Impulsive Behavior Scale (Cyders et al. 2007).

For all statistical programming, the analysis software SAS 9.3 (SAS Institute, Cary, NC) was used. For each parameter estimate, we calculated raw (two sided) $p$-values and 95\% confidence limits. However, since the main focus of this study was based on four hypotheses regarding the influence of ADHD (medicated/unmedicated vs. non-ADHD) on the use of any NUS (yes/no) and the use of multiple different NUS, the (Bonferroni corrected) significance level was considered to be $\alpha=0.05 / 4$, that is, a null hypothesis was rejected for each $p \leq 0.0125$.

\section{Results}

After excluding siblings, 127 children and adolescents with ADHD (51 medicated and 76 unmedicated) and 4940 without ADHD were included in the cohort (Fig. 1).

\section{Sample characteristics}

The male-to-female sex ratio was 4.5:1 in all ADHD subjects and higher in those medicated (Table 1). For both unmedicated and medicated children and adolescents with ADHD, there was a 5-year interval (mean) since the onset of ADHD. Use of non-ADHD prescription drugs was higher in ADHD subjects.

\section{Use of NUS}

Any NUS use was reported by $20 \%$ (26/127) of those with ADHD and by $18 \%$ (877/4940) of those without ADHD (Table 1). Unmedicated subjects with ADHD $(n=76)$ had the highest percentages in almost all categories of NUS. In both groups, with and without ADHD, multivitamin/multimineral complex products were most frequently used. Use of at least two different NUS was 5\% among those without ADHD and 13\% among unmedicated ADHD subjects, while none of the 51 medicated ADHD subjects had use of multiple NUS.

In unmedicated subjects with ADHD who used multiple different NUS $(n=10)$, omega- 3 fatty acids and vitamin D were most frequently used. In subjects without ADHD who used multiple different NUS $(n=235)$, vitamin C was most frequently used (54\%). No melatonin use was reported as an NUS. This was expected as -in contrast to the United States-melatonin is rather uncommon as an NUS in Europe; a $2 \mathrm{mg}$ preparation is even approved as a prescription drug by the European Medicines Agency.

\section{ADHD-dependent differences in using NUS}

Compared with those without ADHD, unmedicated ADHD subjects were more likely to use multiple different NUS (fully adjusted odds ratio [OR], 2.6; 95\% confidence interval [CI], 1.25.6; Table 2). Participants who used non-ADHD prescription drugs 


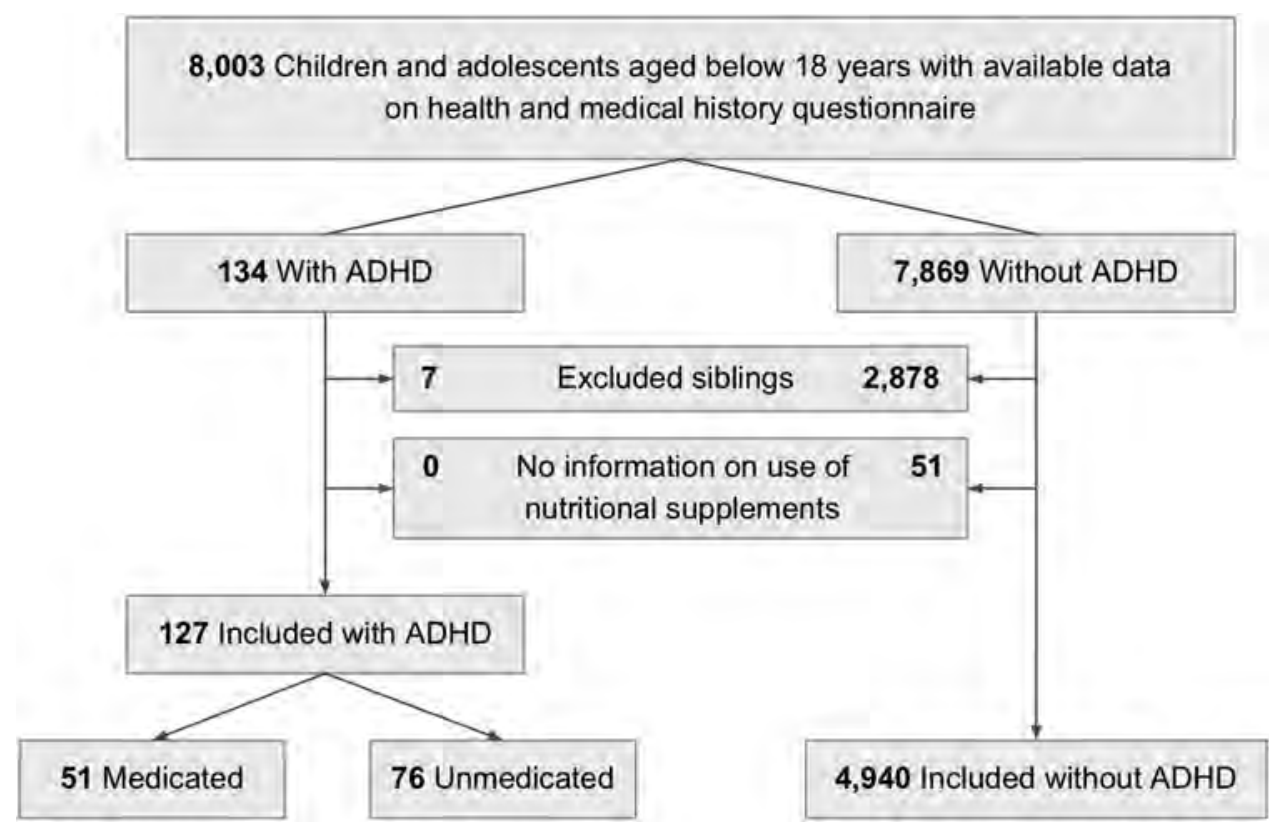

FIG. 1. Participant flow. ADHD, attention-deficit/hyperactivity disorder.

(both ADHD and non-ADHD subjects) were more likely to use any and multiple NUS.

\section{Additional analyses}

Regarding the comparison of all ADHD subjects with nonADHD peers, we found ORs similar to those from the NHANES data (Gardiner et al. 2008). The OR regarding ADHD in our study was 1.2 ; 95\% CI, 0.7-2.0 compared with $1.1 ; 95 \%$ CI, $0.8-1.5$ in Gardiner et al. (2008) and the OR regarding use of prescription drugs was 1.6 ; $95 \%$ CI, 1.2-2.1 compared with 1.4 ; $95 \%$ CI, $1.1-$ 1.7 , respectively.
The comparison of subjects with and without ADHD regarding impulsive behavior revealed that of those with an available score, subjects with ADHD $(n=45)$ had higher scores $(p<0.05)$ than those without $(n=1989)$.

\section{Discussion}

This population-based study found a higher use of multiple different NUS in unmedicated children and adolescents with ADHD compared with medicated ones and those without ADHD. An increased use of any NUS was not statistically significant for both medicated and unmedicated ADHD subjects. Gardiner et al.

Table 1. Sample Characteristics and Nutritional Supplement Use by Group

\begin{tabular}{|c|c|c|c|c|}
\hline & & & $A D H D$ & \\
\hline & 100710 & Unmedicated & Medicated & All cases \\
\hline & $(n=4940)$ & $(\mathrm{n}=76)$ & $(\mathrm{n}=51)$ & $(\mathrm{n}=127)$ \\
\hline Age in years, mean $(\mathrm{SD})$ & $11.6(1.94)$ & $11.7(2.59)$ & $11.4(2.06)$ & $11.6(2.39)$ \\
\hline Male sex, $n(\%)$ & $2454(49.7)$ & $61(80.3)$ & $43(84.3)$ & $104(81.9)$ \\
\hline Years since onset, ${ }^{a}$ mean $(\mathrm{SD})$ & NA & $5(3.2)$ & $5(3.2)$ & $5(3.2)$ \\
\hline Use of non-ADHD prescription drugs, $n(\%)$ & $441(8.9)$ & $10(13.2)$ & $8(15.7)$ & $18(14.2)$ \\
\hline Use of any nutritional supplement within 14 days, $n(\%)$ & $877(17.8)$ & $17(22.4)$ & $9(17.6)$ & $26(20.5)$ \\
\hline Omega-3 fatty acids, $n(\%)$ & $145(2.9)$ & $5(6.6)$ & $1(2.0)$ & $6(4.7)$ \\
\hline Vitamin C, $n(\%)$ & $267(5.4)$ & $5(6.6)$ & $1(2.0)$ & $6(4.7)$ \\
\hline Vitamin $\mathrm{D}, n(\%)$ & $161(3.3)$ & $6(7.9)$ & $0(0.0)$ & $6(4.7)$ \\
\hline Vitamin $\mathrm{B}, n(\%)$ & $20(0.4)$ & $1(1.3)$ & $0(0.0)$ & $1(0.8)$ \\
\hline Multivitamin complex, $n(\%)$ & $162(3.3)$ & $2(2.6)$ & $2(3.9)$ & $4(3.1)$ \\
\hline Multivitamin/multimineral complex, ${ }^{\mathrm{b}} n(\%)$ & $257(5.2)$ & $7(9.2)$ & $4(7.8)$ & $11(8.7)$ \\
\hline Other minerals, $n(\%)$ & $138(2.8)$ & $4(5.3)$ & $0(0.0)$ & $4(3.1)$ \\
\hline Other nutritional supplements, $n(\%)$ & $37(0.7)$ & $2(2.6)$ & $1(2.0)$ & $3(2.4)$ \\
\hline Use of multiple different nutritional supplements from above, ${ }^{\mathrm{c}} n(\%)$ & $235(4.8)$ & $10(13.2)$ & $0(0.0)$ & $10(7.9)$ \\
\hline
\end{tabular}

${ }^{\text {a } B a s e d ~ o n ~ t h o s e ~ u n m e d i c a t e d ~}(n=68)$ and medicated $(n=30)$ with available information.

${ }^{\mathrm{b}}$ More than one vitamin and more than one mineral.

${ }^{c}$ Either simultaneously or sequentially within 14 days. In unmedicated subjects with ADHD who used multiple different nutritional supplements $(n=10)$, omega-3 fatty acids and vitamin $\mathrm{D}$ were most frequently used.

ADHD, attention-deficit/hyperactivity disorder; NA, not applicable; SD, standard deviation. 


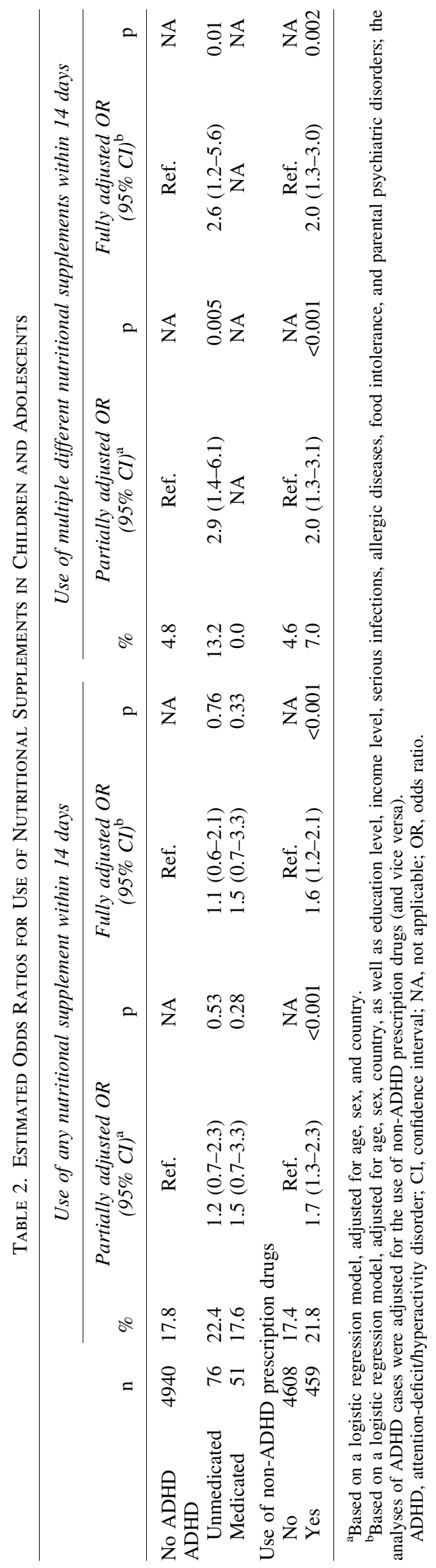

(2008) also used population-based survey data to estimate prevalence of and common factors for use of any NUS. As in our study, not ADHD, but use of prescription drugs was strongly associated with use of any NUS. Similar to our study, multivitamins and vitamin $\mathrm{C}$ were most commonly used underlining the comparability of our results with those from the NHANES data analyzed by Gardiner et al. (2008). The overall prevalence of use of any NUS was, however, higher in their study. This might be because they enquired about use in the prior month, compared with the last 2 weeks in our study.

We hypothesized that the suggested association between ADHD and use of NUS may depend on ADHD medication treatment, since use of prescription drugs in general was a predictor of use of any NUS (Gardiner et al. 2008). In our sample, the point estimate indicated that subjects with medicated ADHD were 50\% more likely to use any NUS; however, due to the small number of children with medicated ADHD, the corresponding wide CI does not allow any reliable conclusions.

Interestingly, we found that use of multiple different NUS in ADHD subjects occurred only in those who were not taking ADHD medication. Consequently, we added this variable as a second measure for the primary outcome, thereby enhancing the reflection of utilization habits. On the one hand, the observed association between use of multiple NUS and unmedicated ADHD was surprising as using prescription drugs in general was a predictor for use of any NUS. On the other hand, this finding is in line with results from Sinha and Efron (2005). They showed that "additional benefit to doctor's treatment" was one of the main reasons $(70 \%)$ to use complementary and alternative medicine in children with ADHD, however, a "benefit in place of doctor's treatment" was still indicated in $30 \%$ of the surveyed parents (Sinha and Efron 2005).

It is possible that we identified-for the first time in a population-based sample — such a subgroup of children and adolescents with ADHD who received parent-initiated self-care interventions with multiple different NUS in place of medication. At first glance, the proportion of $13 \%$ of unmedicated children and adolescents with ADHD who potentially received this intervention appears relatively small. However, given both the epidemiology of ADHD and its correlates and sequelae, it is a notable amount from a public health point of view. Assuming that ADHD medication would have been indicated in these subjects, this therapeutic attempt should be assessed critically.

Unmedicated children and adolescents with ADHD who used multiple different NUS in our study mainly took omega-3 fatty acids and vitamin D. The NICE committee concluded that omega-3 fatty acids had no clinically important benefits for ADHD and, due to lack of data on side effects, that harm could not be excluded (NICE 2018). A later systematic review aimed to examine effects of omega-3 supplementation on cognition in the general population and in those with neurodevelopmental disorders. The authors concluded that there is no evidence for an effect on cognition in both population groups and that advertisement claims of cognitive benefits should be narrowed (Cooper et al. 2015). The same authors concluded in another review that the possibility of moderate to large effects on reducing emotional dysregulation, oppositional behavior, and conduct problems (which commonly accompany ADHD) by omega-3 fatty acid supplementation can be ruled out (Cooper et al. 2016).

However, omega-3 fatty acids might be used as an adjunct therapy to ADHD medication to reduce the dosage (Königs and Kiliaan 2016; Lange et al. 2017), for subjects with subclinical symptoms (Sonuga-Barke 2015), or by families who decline other psychopharmacological options (Bloch and Qawasmi 2011). There 
is hypothetical evidence for the efficacy of vitamin D supplementation in patients with psychiatric disorders on improving brain functions (Patrick and Ames 2015). However, clinical evidence is only available for the effect of vitamin D supplementation on improving ADHD evening symptoms when it is used adjunctively to methylphenidate but not as monotherapy (Mohammadpour et al. 2016).

In addition to the limited evidence on the effectiveness of NUS in unmedicated ADHD patients, consumers in the countries participating in this study usually have to pay for NUS as they are normally not reimbursed by health insurances. This might result in a potentially unnecessary financial burden for families who have a child with ADHD. These families already have a high burden due to the effects of ADHD on family life (Le et al. 2014). Undoubtedly, the generally smaller effect size (Sonuga-Barke et al. 2013; Stevenson et al. 2014; Faraone et al. 2015) of a single administered NUS to treat ADHD, if proven ineffective, should not lead to attempts of enhancing the effect with additional supplements.

As an alternative to combining single conventional NUS, for example, omega-3 fatty acids with vitamin $\mathrm{D}$ as in our study, there is a growing base of literature suggesting that carefully balanced, highly bioavailable multivitamin/multimineral supplements may be beneficial at least for some comorbid children with ADHD (Rucklidge et al. 2018). A recent-first and yet to be confirmedrandomized placebo-controlled study indicated reduction of debilitating symptoms such as emotional dysregulation and aggression by a 48-ingredient micronutrient formula (Rucklidge et al. 2018).

In reviewing the literature, it can be assumed that at least one of three parents does not inform their pediatrician about child's use of complementary and alternative medicine (Chan et al. 2003; Sinha and Efron 2005) and a delay in ADHD treatment due to its use is reportedly common (Vohra et al. 2009; Hurt and Arnold 2014). Research has shown an increased willingness to use medical and psychosocial interventions to treat ADHD among parents and adolescents who felt knowledgeable and among those who considered the treatments acceptable and helpful (Bussing et al. 2012). Hence, the clinical implication of our findings is that pediatricians should actively explore whether parents/patients favor orally administered NUS over medication to treat ADHD core symptoms and, if necessary, emphasize that based on current evidence the average effect size of ADHD medication is larger. In case that families decline psychopharmacological options and prefer NUS, recommendations should optimally be given according to best available evidence.

The main strength of our study lies in the large population-based approach, avoiding clinical sampling biases and improving the statistical certainty of our findings. Another strength is that our data allowed us to differentiate between subjects with medicated and unmedicated ADHD. However, our study has some limitations. First, we did not have information on psychosocial interventions such as behavioral therapy and therefore could only focus on oral remedies (i.e., ADHD medication and NUS). Second, we did not know when the patient had last visited the pediatrician and whether a nutrient deficiency existed possibly justifying use of multiple different NUS. Third, we were not able to assess the severity of ADHD.

Fourth, the calculated prevalence of ADHD in the included sample is somewhat lower than the worldwide prevalence reported in the meta-analysis by Polanczyk et al. (2015). Reasons might be that our prevalence can be considered an administrative prevalence (reported diagnosis), unwillingness to admit a psychiatric disorder in one's child by a parent to the interviewer, and generally lower prevalence rates according to the criteria of the International Classification of Diseases-which is more common in Europethan according to the Diagnostic and Statistical Manual of Mental
Disorders (Döpfner et al. 2008). As the sample of children and adolescents with ADHD is rather small, our interpretations need to be treated with care.

Fifth, parents were only asked for current use of medication. Hence, we do not know whether children who were unmedicated at the time of the study had been medicated in the past. ADHD medication might have not been effective/tolerated and therefore alternative treatments could have been chosen. In addition, we do not know whether unmedicated subjects in our sample benefited from NUS.

Finally, as the study was conducted in several countries, our data were structured hierarchically. Typically, multilevel logistic regression models should be used to counteract false inferences. Our model did not converge for the outcome "use of multiple nutritional supplements." Therefore, we decided on a conventional logistic regression model. However, both models resulted in equivalent findings with the outcome "any nutritional supplement" and research suggests that, given a small number of countries, and when interested in the effect of characteristics on the individual level as in our study, the hierarchical structure can be ignored (Austin et al. 2003; Bryan and Jenkins 2016).

\section{Conclusions}

The results of this study suggest that a notable proportion of unmedicated children and adolescents with ADHD-or presumably rather their parents_-prefer a treatment strategy of using multiple different NUS rather than ADHD medication. As all studied NUS had to be taken orally, this subgroup obviously did not avoid oral remedies in general. The findings in this study add to a growing body of literature on complementary and alternative medicine use in children and adolescents with ADHD suggesting that NUS are potentially taken inappropriately as treatment for ADHD. This study confirmed this fact, for the first time, in a population-based sample and by including a comparison group. Pediatricians should actively enquire in routine care about parentinitiated self-care interventions with NUS at an early stage and provide both the patient and the parent/caregiver with evidencebased patient information about pharmacological and other nonpharmacological ADHD treatments.

\section{Clinical Significance}

This is the first study that showed differences in use of NUS depending on ADHD and medication status in a population-based sample. Unmedicated ADHD children and adolescents are more likely to use multiple different NUS compared with medicated and non-ADHD peers. This finding suggests that a notable proportion of children and adolescents with ADHD or their parents prefer a treatment strategy of taking multiple NUS instead of ADHD medication.

\section{Disclosures}

Dr. Riedel has received unrestricted educational grants from Bayer AG outside the submitted work. Dr. Banaschewski served in advisory or consultancy roles for Actelion, Hexal Pharma, Lilly, Medice, Novartis, Oxford outcomes, PCM scientific, Shire, and Vifor Pharma; received conference support or speaker's fees by Medice, Novartis, and Shire; is/has been involved in clinical trials conducted by Shire and Vifor Pharma; received royalties from Hogrefe, Kohlhammer, CIP Medien, Oxford University Press; declares that the present work is unrelated to the above grants and 
relationships. All other authors report that no competing financial interests exist.

\section{Acknowledgments}

The authors thank the I.Family children and their parents for participating in this extensive examination. They are grateful for the support from school boards, headmasters, and communities. They thank Ina Alvarez (Leibniz Institute for Prevention Research and Epidemiology-BIPS) for her administrative support.

\section{References}

Ahrens W, Bammann K, Siani A, Buchecker K, De Henauw S, Iacoviello L, Hebestreit A, Krogh V, Lissner L, Mårild S, Molnár D, Moreno LA, Pitsiladis YP, Reisch L, Tornaritis M, Veidebaum T, Pigeot I, IDEFICS Consortium: The IDEFICS cohort: Design, characteristics and participation in the baseline survey. Int $\mathrm{J}$ Obes 35:S3-S15, 2011.

Ahrens W, Siani A, Adan R, De Henauw S, Eiben G, Gwozdz W, Hebestreit A, Hunsberger M, Kaprio J, Krogh V, Lissner L, Molnár D, Moreno LA, Page A, Picó C, Reisch L, Smith RM, Tornaritis M, Veidebaum T, Williams G, Pohlabeln H, Pigeot I; on behalf of the I.Family Consortium: Cohort profile: The transition from childhood to adolescence in European children-how I.Family extends the IDEFICS cohort. Int J Epidemiol 46:1394j-1395j, 2017.

Austin PC, Tu JV, Alter DA: Comparing hierarchical modeling with traditional logistic regression analysis among patients hospitalized with acute myocardial infarction: Should we be analyzing cardiovascular outcomes data differently? Am Heart J 145:27-35, 2003.

Bloch MH, Mulqueen J: Nutritional supplements for the treatment of ADHD. Child Adolesc Psychiatr Clin N Am 23:883-897, 2014.

Bloch MH, Qawasmi A: Omega-3 fatty acid supplementation for the treatment of children with attention-deficit/hyperactivity disorder symptomatology: Systematic review and meta-analysis. J Am Acad Child Adolesc Psychiatry 50:991-1000, 2011.

Bryan ML, Jenkins SP: Multilevel modelling of country effects: A cautionary tale. Eur Sociol Rev 32:3-22, 2016.

Bussing R, Koro-Ljungberg M, Noguchi K, Mason D, Mayerson G, Garvan CW: Willingness to use ADHD treatments: A mixed methods study of perceptions by adolescents, parents, health professionals and teachers. Soc Sci Med 74:92-100, 2012.

Chan E, Rappaport LA, Kemper KJ: Complementary and alternative therapies in childhood attention and hyperactivity problems. J Dev Behav Pediatr 24:4-8, 2003.

Cooper RE, Tye C, Kuntsi J, Vassos E, Asherson P: Omega-3 polyunsaturated fatty acid supplementation and cognition: A systematic review and meta-analysis. J Psychopharmacol 29:753-763, 2015.

Cooper RE, Tye C, Kuntsi J, Vassos E, Asherson P: The effect of omega-3 polyunsaturated fatty acid supplementation on emotional dysregulation, oppositional behaviour and conduct problems in ADHD: A systematic review and meta-analysis. J Affect Disord 190:474-482, 2016.

Cyders MA, Smith GT, Spillane NS, Fischer S, Annus AM, Peterson C: Integration of impulsivity and positive mood to predict risky behavior: Development and validation of a measure of positive urgency. Psychol Assess 19:107-118, 2007.

Döpfner M, Breuer D, Wille N, Erhart M, Ravens-Sieberer U; BELLA Study Group: How often do children meet ICD-10/DSM-IV criteria of attention deficit-/hyperactivity disorder and hyperkinetic disorder? Parent-based prevalence rates in a national sample-Results of the BELLA study. Eur Child Adolesc Psychiatry 17:59-70, 2008.

Faraone SV, Asherson P, Banaschewski T, Biederman J, Buitelaar JK, Ramos-Quiroga JA, Rohde LA, Sonuga-Barke EJS, Tannock R,
Franke B: Attention-deficit/hyperactivity disorder. Nat Rev Dis Primers 1:15020, 2015.

Gardiner P, Buettner C, Davis RB, Phillips RS, Kemper KJ: Factors and common conditions associated with adolescent dietary supplement use: An analysis of the National Health and Nutrition Examination Survey (NHANES). BMC Complement Altern Med 8: 9, 2008.

Heilskov Rytter MJ, Andersen LBB, Houmann T, Bilenberg N, Hvolby A, Mølgaard C, Michaelsen KF, Lauritzen L: Diet in the treatment of ADHD in children-A systematic review of the literature. Nord J Psychiatry 69:1-18, 2015.

Hurt EA, Arnold LE: An integrated dietary/nutritional approach to ADHD. Child Adolesc Psychiatr Clin N Am 23:955-964, 2014.

Königs A, Kiliaan AJ: Critical appraisal of omega-3 fatty acids in attention-deficit/hyperactivity disorder treatment. Neuropsychiatr Dis Treat 12:1869-1882, 2016.

Lange KW, Hauser J, Lange KM, Makulska-Gertruda E, Nakamura Y, Reissmann A, Sakaue Y, Takano T, Takeuchi Y: The role of nutritional supplements in the treatment of ADHD: What the evidence says. Curr Psychiatry Rep 19:8, 2017.

Le HH, Hodgkins P, Postma MJ, Kahle J, Sikirica V, Setyawan J, Erder MH, Doshi JA: Economic impact of childhood/adolescent ADHD in a European setting: The Netherlands as a reference case. Eur Child Adolesc Psychiatry 23:587-598, 2014.

Mohammadpour N, Jazayeri S, Tehrani-Doost M, Djalali M, Hosseini M, Effatpanah M, Davari-Ashtiani R, Karami E: Effect of vitamin $\mathrm{D}$ supplementation as adjunctive therapy to methylphenidate on ADHD symptoms: A randomized, double blind, placebo-controlled trial. Nutr Neurosci 7:1-8, 2016.

NICE: Attention deficit hyperactivity disorder: Diagnosis and management. 2018. Available at www.nice.org.uk/guidance/ng87 (accessed April 18, 2018).

Patrick RP, Ames BN: Vitamin D and the omega-3 fatty acids control serotonin synthesis and action, part 2: Relevance for ADHD, bipolar disorder, schizophrenia, and impulsive behavior. FASEB J 29:2207-2222, 2015.

Pliszka S; AACAP Work Group on Quality Issues: Practice parameter for the assessment and treatment of children and adolescents with attention-deficit/hyperactivity disorder. J Am Acad Child Adolesc Psychiatry 46:894-921, 2007.

Polanczyk GV, Salum GA, Sugaya LS, Caye A, Rohde LA: Annual research review: A meta-analysis of the worldwide prevalence of mental disorders in children and adolescents. J Child Psychol Psychiatry 56:345-365, 2015.

Qato DM, Alexander GC, Guadamuz JS, Lindau ST: Prevalence of dietary supplement use in US children and adolescents, 2003-2014. JAMA Pediatr 172:780-782, 2018.

Rucklidge JJ, Eggleston MJF, Johnstone JM, Darling K, Frampton $\mathrm{CM}$ : Vitamin-mineral treatment improves aggression and emotional regulation in children with ADHD: A fully blinded, randomized, placebo-controlled trial. J Child Psychol Psychiatry 59:232-246, 2018.

Sinha D, Efron D: Complementary and alternative medicine use in children with attention deficit hyperactivity disorder. J Paediatr Child Health 41:23-26, 2005.

Sonuga-Barke EJS: Editorial: Diet and children's behaviour problems-Disentangling urban myth from clinical reality. J Child Psychol Psychiatry 56:497-499, 2015.

Sonuga-Barke EJS, Brandeis D, Cortese S, Daley D, Ferrin M, Holtmann M, Stevenson J, Danckaerts M, van der Oord S, Döpfner M, Dittmann RW, Simonoff E, Zuddas A, Banaschewski T, Buitelaar J, Coghill D, Hollis C, Konofal E, Lecendreux M, Wong ICK, Sergeant J; European ADHD Guidelines Group: Nonpharmacological interventions for ADHD: Systematic review and 
meta-analyses of randomized controlled trials of dietary and psychological treatments. Am J Psychiatry 170:275-289, 2013.

Stevenson J, Buitelaar J, Cortese S, Ferrin M, Konofal E, Lecendreux M, Simonoff E, Wong ICK, Sonuga-Barke EJS: Research review: The role of diet in the treatment of attention-deficit/hyperactivity disorder-An appraisal of the evidence on efficacy and recommendations on the design of future studies. J Child Psychol Psychiatry 55:416-427, 2014.

Subcommittee on Attention-Deficit/Hyperactivity Disorder; Steering Committee on Quality Improvement and Management, Wolraich M, Brown L, Brown RT, DuPaul G, Earls M, Feldman HM, Ganiats TG, Kaplanek B, Meyer B, Perrin J, Pierce K, Reiff M, Stein MT, Visser S: ADHD: Clinical practice guideline for the diagnosis, evaluation, and treatment of attention-deficit/hyperactivity disorder in children and adolescents. Pediatrics 128:1007-1022, 2011.

Taylor E, Döpfner M, Sergeant J, Asherson P, Banaschewski T, Buitelaar J, Coghill D, Danckaerts M, Rothenberger A, SonugaBarke EJS, Steinhausen H-C, Zuddas A: European clinical guidelines for hyperkinetic disorder-First upgrade. Eur Child Adolesc Psychiatry 13:i7-i30, 2004.

United Nations Educational, Scientific and Cultural Organisation: International Standard Classification of Education (ISCED). 2011.
Available at http://uis.unesco.org/sites/default/files/documents/ international-standard-classification-of-education-isced-2011-en .pdf (accessed April 18, 2018).

Visser SN, Danielson ML, Bitsko RH, Perou R, Blumberg SJ: Convergent validity of parent-reported attention-deficit/hyperactivity disorder diagnosis: A cross-study comparison. JAMA Pediatr 167: 674-675, 2013.

Vohra S, Brulotte J, Le C, Charrois T, Laeeque H: Adverse events associated with paediatric use of complementary and alternative medicine: Results of a Canadian Paediatric Surveillance Program survey. Paediatr Child Health 14:385-387, 2009.

Address correspondence to:

Hermann Pohlabeln, PhD

Department of Biometry and Data Management Leibniz Institute for Prevention Research and Epidemiology-BIPS 28359 Bremen Germany

E-mail: pohlabeln@leibniz-bips.de 